\title{
Second Primary Malignancies in Pediatric and Adolescent Nasopharyngeal Carcinoma Survivors: Experience of a Single-center
}

\author{
(1) Sema BÜYÜKKAPU BAY,' (1) Kübra ÖZKAYA, ${ }^{2}$ (D) Rejin KEBUDi,' (1) Musa ALTUN² \\ 'Department of Pediatric Hematology-Oncology, Istanbul University Oncology Institute, Istanbul-Turkey \\ ${ }^{2}$ Department of Radiation Oncology, Istanbul University Oncology Institute, Istanbul-Turkey
}

\begin{abstract}
OBJECTIVE
The aim of this study is to investigate the incidence and clinicopathologic characteristics of second primary malignancies (SPMs) in pediatric and adolescent nasopharyngeal carcinoma (NPC) survivors from the point of patients' age, emergence time, location and histopathology.
\end{abstract}

\section{METHODS}

Medical reports of pediatric NPC patients (patients at or under the age of 19) treated between 1990 and 2017 and followed till the end 2020 were reviewed. SPMs are classified depending on the organ, histopathology, location (whether in or out of the radiation field). Additionally time period between NPC treatment and diagnosis of second primaries are recorded.

\section{RESULTS}

The median age of the 108 patients at diagnosis was 15 years (5-19 years). The male-to-female ratio was 2.1. The median follow-up was 118 months (3-332 months). Eight patients developed 9 second malignancies (one patient with two SPMs). Eight of SPMs were in the radiation field. SPMs developed at a median of 15 years (5-26 years) after the conclusion of the primary treatment. Among patients, whose malignancy developed in the irradiation field 6 were treated primarily with complete surgical resection and 3 of them died because of SPM.

\section{CONCLUSION}

Incidences of SPMs have been increasing in parallel with the increase in life expectancy of pediatric and adolescent NPC survivors. Due to relatively high numbers of SPMs, pediatric/adult nasopharyngeal cancer survivors should be followed regularly not only for recurrences and long term morbidities but also for second malignancies which may be treated with curative surgeries and additional treatments when diagnosed early. The patient follow-up should continue and patients should be evaluated comprehensively, taking into account cancers outside the irradiation field. Since the most of the SPMs develops in the irradiation field, prospective clinical studies investigating dose reduction in the treatment of pediatric NPC should be considered and evaluated with further prospective trials.

Keywords: Adolescent; children; nasopharyngeal carcinoma; second primary malignancies. Copyright $\odot$ 2021, Turkish Society for Radiation Oncology 


\section{Introduction}

Survival rates of childhood/adolescent cancers have risen from $10 \%$ to almost $85 \%$ in the past 60 years with more efficient and less toxic treatment protocols.[1] These high survival rates also mean a growing population of younger survivors who will live with treatment sequelae for a long time. Second and/or secondary malignancies are among the upmost important long-term health problems encountered by cancer survivors.[2] Second primary malignancies (SPMs) are the primary mortality cause in the long term for individuals with two primary cancers. [3] Hence, prevention and early detection of SPM is critical for prolonging life for cancer survivors.

Pediatric/adolescent cancer survivors have an increased risk of developing subsequent primary neoplasms, which is estimated to be 10 times higher than the general population and may occur due to the carcinogenic influences of radiotherapy (RT), chemotherapeutic agents, environmental carcinogens, and genetic features.[4] Squamous cell carcinoma (SCC) histology is very rare among pediatric/adolescent head-and-neck tumors, and nearly all of them are nasopharyngeal carcinoma (NPC). NPC has a bimodal age distribution around the Mediterranean basin with the first peak between 10 and 20 years of age.[5] Childhood/adolescent undifferentiated NPC (type III) is generally associated with Epstein-Barr virus infection and is very sensitive to radiation.[5]

High-level clinical evidence for the management of pediatric and adolescent patient populations is lacking. Current patient evaluation and treatment protocols are mostly derived from adult head-and-neck SCC protocols, which rely on the adult patient population's clinical data.[6,7] Ultimately pediatric/adolescent NPC treatment comprises induction chemotherapy and high-dose definitive RT combination. Because of critical structures in the vicinity and relatively high therapeutic doses, RT of NPC may increase the rate of late toxicities.

There are limited data on the incidence of SPMs among pediatric and young NPC survivors. [8-14] This study aimed to investigate the incidence and clinicopathologic characteristics of SPMs among survivors pediatric and adolescent NPC patients treated between 1990 and 2017.

\section{Materials and Methods}

From the patient records, NPC patients at or under the age of 19 treated between 1990 and 2016 and followed until 2020 were retrospectively identified. The included cases were biopsy-proven, previously untreated NPC patients treated with curative intent using induction chemotherapy followed by RT. One hundred and eight cases were identified and reviewed for the treatment details, outcomes, and incidence of second malignancies. The patient assessments at diagnosis included physical and endoscopic examination, laboratory studies, and head-and-neck radiological examination performed with magnetic resonance imaging (MRI) and/ or computed tomography (CT). Tumor staging at initial diagnosis included chest CT and bone scintigraphy until 2006, and thereafter, fluorodeoxyglucose positron emission tomography-CT (PET-CT) was performed. Tumors were staged according to the $7^{\text {th }}$ edition of the American Joint Cancer Committee TNM staging system.[15] All statistical analyses were performed by SPSS 10.0 (SPSS Inc., Chicago, IL). The local ethics committee approved the study.

\section{Treatment}

The primary treatment was three courses of chemotherapy followed by RT. Patients diagnosed during 1989-1991 were treated with cisplatin $\left(80 \mathrm{mg} / \mathrm{m}^{2}\right)-5$ fluorouracil $\left(1000 \mathrm{mg} / \mathrm{m}^{2} / \mathrm{d}\right.$ on days $\left.1-4\right)$ combination, during 1992-2007 with cisplatin $\left(100 \mathrm{mg} / \mathrm{m}^{2} /\right.$ day), epirubicin $\left(90 \mathrm{mg} / \mathrm{m}^{2} /\right.$ day $)$, and bleomycin $\left(15 \mathrm{mg} / \mathrm{m}^{2} /\right.$ day) combination and since 2008 , cisplatin $(100 \mathrm{mg} /$ $\mathrm{m}^{2} /$ day) and epirubicin $\left(90 \mathrm{mg} / \mathrm{m}^{2} /\right.$ day $)$ combination were administered with 3-week intervals. RT was administered without concurrent chemotherapy.

All patients received external beam RT. Before 2011, conventional RT with two-dimensional treatment planning was used. The primary tumor and upper neck area were treated with two parallel opposed lateral fields, the lower cervical and supraclavicular regions were treated by a single anterior field using a median shield to protect the larynx and the spinal cord, with doses of $50 \mathrm{~Gy}$ by Co60 or 4-6 MV linear accelerator. The spinal cord was shielded after $46 \mathrm{~Gy}$ and the posterior lymphatic chains were treated with electron beams. A total median dose of 66 Gy (range 60-70 Gy) was administered in daily fractions of 1.8-2 Gy, 5 days a week for all the primary tumor and involved lymph nodes. Uninvolved cervical and supraclavicular regions received 45-50 Gy in daily fractions of 1.8-2 Gy, 5 days a week. Since 2011, intensity-modulated RT (IMRT) was used. The gross tumor volume (GTV) included the whole extent of disease in the primary nasopharyngeal lesion and cervical lymph nodes observed on magnetic resonance and/ or CT images. Accordingly, the clinical target volume (CTV) included all GTV with safety margins covering 
all potential subclinical disease sites; posterior nasal cavity, maxillary sinuses, clivus, basis cranii, inferior sphenoid sinus, pterygoid fossae, and retropharyngeal nodes with $0.5-1 \mathrm{~cm}$ margins. The main critical organs to be spared were the parotid glands, pituitary gland, cochlea, brain stem, spinal cord, larynx, eyes, lens, chiasma, optic nerves, and brachial plexus. Planning target volume was obtained by an additional $0.3-0.5 \mathrm{~cm}$ around CTVs.

Post-treatment imaging was performed by CT or MRI at 6-8 weeks and by PET-CT at 12 weeks after completion of the therapy. The periods for followup examinations were once in 3 months for the first 2 years after RT, every $4-6$ months for the $3^{\text {rd }}, 4^{\text {th }}$, and $5^{\text {th }}$ years, and annually thereafter. During follow-up, imaging surveillance by CT or MRI was held every 6 months for the first 2 years and annually thereafter. PET-CT imaging was performed in case of clinical necessity. Histopathologic confirmation was obtained for diagnosis of second primary cancer. Locoregional $\mathrm{MRI} / \mathrm{CT}$ and/or PET-CT were used for second primary cancer evaluation.

\section{Results}

At diagnosis, the median age of the 108 pediatric/adolescent NPC patients was 15 years (5-18 years). The male-to-female ratio was 2.1 and 53 (49\%) patients were younger than 14 years of age. The main histological type was the World Health Organization (WHO) type III undifferentiated carcinoma. Eighty-six (79.6\%) had WHO type III, $21(19.4 \%)$ had WHO type II (nonkeratinized), and 1 (1\%) had WHO type 1 (keratinized) carcinoma. Four (3.7\% of patients had Stage II disease, the remaining 104 (96.3\%) had Stages III and IV disease at presentation.

All patients were treated with cisplatin-based three courses of neoadjuvant chemotherapy by every 3 weeks interval followed by RT. Chemotherapy regimens used were cisplatin, 5-fluorouracil in 11 patients (90\%) before 1992, cisplatin, epirubicin with or without bleomycin in 97 patients (90\%) later-on. Eighty-one patients (75\%) were treated with 2D-RT and $27(25 \%)$ patients with IMRT. The median follow-up was 118 months (3-332 months).

In $15(14 \%)$ patients, the disease continued and/or relapsed at a median of 8 months (range, 2-23 months). The failure sites were distant in 10 cases, distant and regional in three cases, distant and local one case, and distant and local and regional in one case.

During follow-up 21 patients died, 15 of them died due to progressive disease, three from the second malignancy, and the rest three deaths were unrelated to malignancy.

\section{Second Malignancies}

In eight patients, nine SPMs developed (a patient had two SPMs). Excluding the SPM in the bladder, all were in the irradiation field (Table 1). In all the patients with SPM, SPM developed at a median of 15 years (526 years) after the end of initial treatment. The mean duration between the first and second primary cancer diagnoses was 19 years for those at/or above 14 years of age and only 5 years for those under the age of 14 .

The site of SPM in our patients showed a significant risk of the oral cavity and pharyngeal cancers, which made up three of nine SPMs. Three of the nine SPMs were sarcomas (one osteosarcoma, one fibrosar-

Table 1 Clinical characteristics in patients with second malignancies

\begin{tabular}{|c|c|c|c|c|c|}
\hline Second malignancy & Gender & $\begin{array}{l}\text { Age at diagnosis } \\
\text { of NPC (years) }\end{array}$ & $\begin{array}{c}\text { Treatment } \\
\text { schedule at } \\
\text { first diagnosis }\end{array}$ & $\begin{array}{c}\text { Duration of } \\
\text { occurrence of second } \\
\text { malignancy (years) }\end{array}$ & Outcome \\
\hline Osteosarcoma of the mandible & M & 12 & CT, RT (70 Gy) & 5 & DwD (4 years) \\
\hline PNET in bladder & M & 11 & CT, RT (66 Gy) & 5 & DwD (2 years) \\
\hline Thyroid papillary Ca & $\mathrm{F}$ & 13 & CT, RT (66 Gy) & 12 & NED (5 years) \\
\hline Tonsil, base of tongue carcinoma & M & 15 & CT, RT (66 Gy) & 20 & DwD (1 year) \\
\hline Hypopharynx carcinoma & M & 17 & CT, RT (70 Gy) & 14 & NED (6 years) \\
\hline Nasal cavity carcinoma & M & 17 & CT, RT (70 Gy) & 12 & NED (15 years) \\
\hline \multirow[t]{2}{*}{ Fibrosarcoma carcinoma locally } & M & 17 & CT, RT (70 Gy) & 18 & NED (8 years) \\
\hline & & 17 & & 25 & NED (3 years) \\
\hline Esophagus proximal carcinoma & M & 14 & CT, RT (68 Gy) & 26 & DwD (1 year) \\
\hline
\end{tabular}

NPC: Nasopharyngeal carcinoma; PNET: Primitive neuroectodermal tumor; DwD: Died with disease; NED: Alive with no evidence of disease; RT: Radiotherapy; M: Male; F: Female; Ca: Carcinoma; CT: Chemotherapy 
coma, and one high-grade sarcoma of the temporal fossa) and five of the nine SPMs were carcinomas. The patient with a SPM in his bladder was diagnosed with primitive neuroectodermal tumor (PNET), he received chemotherapy and $\mathrm{RT}$, however, he died because of the progression of the second SPM. The other seven patients, whose second malignancies developed in the irradiation field, underwent curative surgeries; four are alive with no evidence of disease (NED) for a median of 7 years (3-15 years), one, who was with NED for 4 years, died from sepsis after reconstructive surgery of the mandible. Three of the patients died from progressive SPMs.

\section{Discussion}

This report is one of the largest data on SPMs in pediatric/adolescent NPC survivors. We identified 8 (7.4\%) patients with SPMs among 108 NPC patients. In adult patients, there have been population-based studies about the risk of SPM after NPC treatment in different countries,[16-19] but in pediatric and adolescence NPC patients, SPM has been described in the longterm morbidities of childhood/adolescent carcinoma treatment by the centers' experience.[8-14,20,21]

Among pediatric head-and-neck cancer patients, the male gender was more prevalent in most studies, accounting for approximately $60 \%$ of the primary cases[22] with similar to NPC patients in different centers. [9-12] The female gender was more predominant among primary thyroid carcinoma patients.[22] In our study, the male-to-female ratio in all pediatric/adolescent NPC patients was 2.1:1 while it was higher in SPM patients $(7: 1)$.

Adult female NPC survivors had a higher risk of SPM than adult males in every interval[16,17] although most studies on young NPC patients did not report a difference in incidence regarding sex. $[9,10,12,13]$ In the report of Jouin et al.,[11] two female patients; in Schmidt et al.[8] reported one female and one male patient; and in Cağlar et al.[14] reported one male patient with NPC developed a second malignancy. No definite conclusion can be drawn for a gender predominance on SPMs in pediatric and adolescent NPC survivors since no appropriate study groups exist.

The mean duration after the conclusion of the primary treatment to the diagnosis of SPM was 5 years for the younger patients (younger than age of 14) and 19 years for the older (14-18 years of age) patients. Jouin et al.[11] reported two cases of SPM among 95 patients. The first one was 13 years old at diagnosis and developed SPM 6 years after treatment. The second one was 17 years old at the diagnosis and developed SPM 8 years after the treatment. The Denmark study reported two patients with SPMs; a 14-year-old patient developed a SPM at 7 years and a 16-year-old patient developed a SPM at 19 years after the initial treatment.[8] A population-based adult NPC study from Taiwan estimated the cumulative risks of second primaries; 5, 10, 15 , and 20 years after NPC diagnosis as $1.89 \%, 3.26 \%$, $4.23 \%$, and $5.37 \%$, respectively.[17] We observed that the time interval with index tumor treatment and the development of SPM was longer in the relatively older pediatric NPC survivors.

Population-based studies on adult patients have shown that the SPM incidence was higher for patients diagnosed before 40 years of age than later age.[16-18] Most reports on SPMs in pediatric/adolescent NPC survivors did not compare age groups with each other. $[9,10,12,13]$ Even after an evaluation with former studies, $[8,11]$ our data are not sufficient to conclude that younger pediatric NPC patients have more risk for developing subsequent SPM than older pediatric/adolescent NPC patients conclusively.

Radiation-induced tumors are the most serious late complications in NPC survivors and the incidence rate in adult patients was reported to be between $0.1 \%$ and 5.6\%.[17-19,23] Multiple epidemiological studies have confirmed the importance of age at radiation exposure. The younger are more susceptible to radiation than the older.[24,25] Reports on RT-induced tumors point out a large range of histological subtypes. $[8-11,13,14]$

The population-based studies in adult NPC survivors have revealed excess head-and-neck SPMs within the radiation field. The most common SPMs in this region are sarcomas, oral-oropharyngeal carcinomas, and non-melanoma skin cancers.[16-18,26] The data on pediatric/adolescent patients have come from singlecenter reports or pooled data with adult patients from a high incidence area. Single-center reports from various countries about the site and type of SPM in pediatric/ adolescent NPC are SCC of the oral cavity in two patients (Jouin et al.),[11] non-melanoma skin carcinoma in two patients (Schmidt et al.), [8] and osteosarcoma, fibrosarcoma, testicular high-grade sarcoma, and chronic myeloid lymphoma in four patients (Ben-Ami et al.).[9]

Our patients showed a significant risk of the oral cavity and pharyngeal cancers, which made up three of nine SPMs. Three of the nine SPMs were sarcomas (one osteosarcoma, one fibrosarcoma, and one highgrade sarcoma). Overall, eight of the nine SPMs were in the radiation field. 
There is an uncertainty in estimating the exact incidence of radiation-induced SPM because of the confounding factors such as patient lifestyle, genetic abnormalities, and received systemic chemotherapy.[27]

Among our eight SPM patients, whose malignancy developed in the irradiation field, six were treated primarily with complete surgical resection and three of them died of SPM.

Diagnosis of SPM in an early stage and treatment with surgical resection is important in survival. Physicians should be cautious about second neoplasia risk in radiation fields during follow-up and able to detect early in pediatric NPC survivors.[19,28]

\section{Conclusion}

Multimodal treatments lead to high survival and locoregional control rate in childhood-adulthood NPC. In parallel with treatment advancements, SPMs are among the primary challenges. Treatment and follow-up guidelines based on high-level clinical evidence for this group of patients are still lacking. Due to relatively high numbers of SPMs, pediatric/adult nasopharyngeal cancer survivors should be followed regularly not only for recurrences and long-term morbidities but also for second malignancies which may be treated with curative surgeries and additional treatments when diagnosed early. The patient followup should continue and patients should be evaluated comprehensively, taking into account cancers outside the irradiation field.

Since the most of the SPMs develop in the irradiation field, prospective clinical studies investigating dose reduction in the treatment of pediatric NPC should be considered and evaluated with further prospective trials.

Peer-review: Externally peer-reviewed.

Conflict of Interest: All authors declared no conflict of interest.

Ethics Committee Approval: The study was approved by the Local Ethics Committee of Istanbul University Oncology Institute (No: 186362, Date: 27/04/2021).

Financial Support: None declared.

Authorship contributions: Concept - S.B.B., R.K., M.A.; Design - S.B.B., K.Ö., R.K., M.A.; Supervision - S.B.B., K.Ö., R.K., M.A.; Funding - None; Materials - S.B.B., K.Ö., R.K., M.A.; Data collection and/or processing - S.B.B., K.Ö., M.A.; Data analysis and/or interpretation - S.B.B., K.Ö., R.K., M.A.; Literature search - S.B.B., K.Ö., M.A.; Writing - S.B.B., K.Ö., M.A.; Critical review - M.A.

\section{References}

1. O'Leary M, Krailo M, Anderson JR, Reaman GH; Children's Oncology Group. Progress in childhood cancer: 50 years of research collaboration, a report from the Children's Oncology Group. Semin Oncol 2008;35(5):484-93.

2. Wood ME, Vogel V, Ng A, Foxhall L, Goodwin P, Travis LB. Second malignant neoplasms: assessment and strategies for risk reduction. J Clin Oncol 2012;30(30):3734-45.

3. Donin N, Filson C, Drakaki A, Tan HJ, Castillo A, Kwan L, et al. Risk of second primary malignancies among cancer survivors in the United States, 1992 through 2008. Cancer 2016;122()19:3075-86.

4. Neglia JP, Friedman DL, Yasui Y, Mertens AC, Hammond S, Stovall M, et al. Second malignant neoplasms in five-year survivors of childhood cancer: Childhood cancer survivor study. J Natl Cancer Inst 2001;93(8):618-29.

5. Ayan I, Altun M. Nasopharyngeal carcinoma in children: Retrospective review of 50 patients. Int J Radiat Oncol Biol Phys 1996;35(3):485-492.

6. Blanchard P, Lee A, Marguet S, Leclercq J, Ng WT, $\mathrm{Ma}$ J, et al. Chemotherapy and radiotherapy in nasopharyngeal carcinoma: An update of the MAC-NPC meta-analysis. Lancet Oncol 2015;16(6):645-55.

7. Chen YP, Ismaila N, Chua ML, Colevas AD, Haddad $\mathrm{R}$, Huang $\mathrm{SH}$, et al. Chemotherapy in combination with radiotherapy for definitive-intent treatment of stage II-IVA nasopharyngeal carcinoma: CSCO and ASCO guideline. J Clin Oncol 2021;39(7):840-59.

8. Jensen JS, Grønhøj C, Kjær EK, Charabi BW, von Buchwald C, Hjuler T. Second primary cancers in pediatric head and neck cancer survivors in Denmark during 1980-2014: A nationwide study. Int J Pediatr Otorhinolaryngol 2019;127:109648.

9. Ben-Ami T, Ash S, Ben-Harosh M, Gavriel H, Weintraub M, Revel-Vilk S, et al. Nasopharyngeal carcinoma in children and young adults-Beyond 5-year survival. Pediatr Blood Cancer 2020;67(9):e28494.

10. Cheuk DK, Billups CA, Martin MG, Roland CR, Ribeiro RC, Krasin MJ, et al. Prognostic factors and long-term outcomes of childhood nasopharyngeal carcinoma. Cancer 2011;117(1):197-206.

11. Jouin A, Helfre S, Bolle S, Claude L, Laprie A, Bogart E, et al. Adapted strategy to tumor response in childhood nasopharyngeal carcinoma: The French experience. Strahlenther Onkol 2019;195(6):504-16.

12. Lu S, Chang H, Sun X, Zhen Z, Sun F, Zhu J, et al. Long-term outcomes of nasopharyngeal carcinoma in 148 children and adolescents. Medicine (Baltimore) 2016;95(17):e3445.

13. Kontny U, Franzen S, Behrends U, Bührlen M, Chris- 
tiansen H, Delecluse H, et al. Diagnosis and treatment of nasopharyngeal carcinoma in children and adolescents recommendations of the GPOH-NPC study group. Klin Padiatr 2016;228(3):105-12.

14. Cağlar K, Varan A, Akyüz C, Selek U, Kutluk T, Yalçin $B$, et al. Second neoplasms in pediatric patients treated for cancer: A center's 30-year experience. J Pediatr Hematol Oncol 2006;28(6):374-8.

15. Edge SB, Byrd DR, Compton CC, Fritz AG, Greene FL, Trotti A, editors. AJCC Cancer Staging Manual. $7^{\text {th }}$ ed. New York: Springer; 2010.

16. Chan JY, Gooi Z, Mydlarz WK, Agrawal N. Risk of second primary malignancy after nasopharyngeal carcinoma in the United States: A population-based study. Head Neck 2016;38(Suppl 1):E1130-6.

17. Chen MC, Feng IJ, Lu CH, Chen CC, Lin JT, Huang $\mathrm{SH}$, et al. The incidence and risk of second primary cancers in patients with nasopharyngeal carcinoma: A population-based study in Taiwan over a 25 -year period (1979-2003). Ann Oncol 2008;19(6):1180-6.

18. Scélo G, Boffetta P, Corbex M, Chia KS, Hemminki $\mathrm{K}$, Friis $\mathrm{S}$, et al. Second primary cancers in patients with nasopharyngeal carcinoma: A pooled analysis of 13 cancer registries. Cancer Causes Control 2007;18(3):269-78.

19. Lou J, Jiang L, Dai X, Wang H, Yang J, Guo L, et al. Radiation-induced sarcoma of the head and neck following radiotherapy for nasopharyngeal carcinoma: A single institutional experience and literature review. Front Oncol 2021;10:526360.

20. Scholz-Kreisel P, Kaatsch P, Spix C, Schmidberger H, Marron M, Grabow D, et al. Second malignancies fol- lowing childhood cancer treatment in Germany from 1980 to 2014. Dtsch Arztebl Int 2018;115(23):385-92.

21. Marti JL, Jain KS, Morris LG. Increased risk of second primary malignancy in pediatric and young adult patients treated with radioactive iodine for differentiated thyroid cancer. Thyroid 2015;25(6):681-7.

22. Arboleda LPA, de Mendonça RM, Lopez EE, Araújo AL, Palmier NR, de Pauli Paglioni M, et al. Global frequency and distribution of head and neck cancer in pediatrics, a systematic review. Crit Rev Oncol Hematol 2020;148:102892.

23. Liu C, Liao L, Wu G, Yan H, Chen X, Wang C, et al. Radiation-induced second primary squamous cell carcinoma of the oral cavity after radiotherapy for nasopharyngeal carcinoma. Oral Oncol 2020;109:104863.

24. Hall EJ. Intensity-modulated radiation therapy, protons, and the risk of second cancers. Int J Radiat Oncol Biol Phys 2006;65(1):1-7.

25. International Commission on Radiological Protection. Recommendations. Ottawa, Canada: Annals of the ICRP Publication 60; 1990.

26. Chow JC, Au KH, Mang OW, Cheung KM, Ngan RK. Risk, pattern and survival impact of second primary tumors in patients with nasopharyngeal carcinoma following definitive intensity-modulated radiotherapy. Asia Pac J Clin Oncol 2019;15(1):48-55.

27. Kumar S. Second malignant neoplasms following radiotherapy. Int J Environ Res Public Health 2012;9(12):4744-59.

28. Altun M, Tambas M, Dagoglu N. Radiation Infield Second Tumors in Nasopharyngeal Carcinoma Patients. Abstract P2791, s523, ASTRO; 2014. 